\title{
Neue Therapieoption für Kataplexie bei Narkolepsie
}

Fragestellung: Ist Pitolisant, ein inverser $\mathrm{H}_{3}$-Rezeptor-Agonist, der die histaminerge Neurotransmission verstärkt, eine sichere und effektive Therapie für Kataplexie bei Narkolepsie im Vergleich mit Placebo?

Hintergrund: Narkolepsie ist gekennzeichnet durch eine exzessive Tagesmüdigkeit, sleep-onset REM-Schlaf, kataplektische Anfälle, hypnagoge Halluzinationen und Schlaflähmungen. Es finden sich ein Mangel an orexinproduzierenden Nervenzellen im lateralen Hypothalamus und eine vermehrte Anzahl histaminerger tubero-mamillärer Neurone bei normalen Histaminwerten im Liquor. Die Autoren überprüften die Hypothese, dass eine verstärkte histaminerge Neurotransmission das Orexindefizit ausgleichen könnte. Es gibt bisher nur zwei Behandlungsmöglichkeiten für die Kataplexie bei Narkolepsie: Trizyklika, die off-label verwendet werden, und Oxybat. Pitolisant zeigte sich bereits gegen die Tagesmüdigkeit bei Narkolepsie als wirksam, und es ergaben sich Anhaltspunkte in einer Post-hoc-Analyse dieser Studie, dass es auch gegen die Kataplexie wirksam sein könnte [1].

Patienten und Methodik: Patienten $\geq 18$ Jahre mit einer Narkolepsie mit Kataplexie, die durch einen pathologischen Multiplen Schlaf-Latenz-Test (MSLT) mit verkürzter Ein-

Szakacs Z, Dauvilliers Y, Mikhaylov $V$ et al. Safety and efficacy of pitolisant on cataplexy in patients with narcolepsy: a randomised, double-blind, placebo-controlled trial. Lancet Neurol 2017; 16: 200-7 schlaflatenz und sleep-onset REM diagnostisch gesichert wurden, wurden in die Studie eingeschlossen, wenn sie mindestens drei kataplektische Anfälle pro Woche und eine exzessive Tagesmüdig- keit aufwiesen (Epworth Sleepiness Scale score, ESS $\geq 12$ ). Sie wurden 1:1 randomisiert in den Verum- oder Placeboarm eingeschlossen. Nach einer Baseline von zwei Wochen konnte die Dosis von Pitolisant über weitere drei Wochen zwischen einer Dosis von $5 \mathrm{mg}$ und $40 \mathrm{mg}$ in einmal täglicher Gabe angepasst werden, die dann über vier Wochen stabil gehalten wurde. Innerhalb einer weiteren Woche wurden Verum oder Placebo wieder vollständig abgesetzt.

Ergebnisse: In den Verumarm wurden 54 Patienten eingeschlossen, 51 Patienten erhielten Placebo (ITT-Population). Die wöchentliche Anzahl kataplektischer Anfälle (WCR) in der stabilen Dosierungsphase verringerte sich in der Verumgruppe um $75 \%$ und um $38 \%$ in der Placebogruppe $(\mathrm{p}<0,0001)$. Auch die einzelnen Dosissubgruppen (10 mg: $n=7,20 \mathrm{mg}$ : $n=9$, $40 \mathrm{mg}$ : $\mathrm{n}=35$ ) unterschieden sich jeweils signifikant gegenüber Placebo. Zudem waren fast alle sekundären Endpunkte ebenfalls signifikant zugunsten von Pitolisant (z. B. relative Anzahl der Patienten mit WCR $>15$, mittlerer Abfall im ESS, relative Anzahl der Patienten mit ESS $\leq 10$, maintenance of wakefulness test, Anzahl der Episoden mit Halluzinationen).

Behandlungsassoziierte Nebenwirkungen waren in der Verumgruppe deutlich häufiger ( $28 \%$ vs. $12 \%$, p =0,048). Diese umfassten Kopfschmerzen, Irritabilität, Angst und Übelkeit und waren von milder bis mittlerer Intensität. Schwere Nebenwirkungen traten nicht auf. Es gab keine Entzugssymptomatik nach Absetzen in der Verum- und einen solchen Fall in der Placebogruppe.

Schlussfolgerungen: Die Gabe von Pitolisant reduzierte die Anzahl von kataplektischen Anfällen und zahlreichen anderen $\mathrm{Pa}$ rametern (auch die Tagesmüdigkeit und Halluzinationen) signifikant gegenüber Placebo bei guter Verträglichkeit.

\section{- Kommentar von Holger Lerche, Tübingen}

\section{Hoffnungsvolle Therapieoption für Narkolepsiepatienten}

Diese randomisierte, doppelblinde, placebokontrollierte Studie zeigt sehr deutliche Effekte von Pitolisant auf die Kataplexie und andere relevante Parameter bei Narkolepsie. Neben Oxybat wäre es ein weiteres Medikament, das wahrscheinlich auf alle Symptome der Narkolepsie eine gute Wirkung hat. Beim Absetzen zeigte sich zudem keine Entzugssymptomatik und das Nebenwirkungsprofil erscheint günstig. Der genaue Wirkmechanismus von Pitolisant bedarf weiterer Aufklärung. Die Placeborate war sehr hoch, was jedoch für die emotionsabhängigen Symptome bei Narkolepsie typisch ist. Zusammenfassend ist dies eine überzeugende Studie, die eine neue, hoffnungsvolle Therapieoption für Narkolepsiepatienten aufzeigt.

\author{
Referenz \\ 1. Dauvilliers Y et al. Lancet Neurol 2013; 12: $1068-75$
}

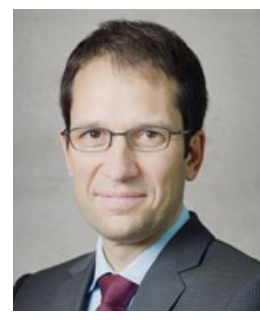

Prof. Dr. med. Holger Lerche, Tübingen

Universitätsklinikum Tübingen, Hertie Institut für Klinische Hirnforschung, Neurologie mit Schwerpunkt Epileptologie E-Mail: holger.lerche@uni-tuebingen.de 